\title{
Endoscopic sphincterotomy using a pull-type sphincterotome with an attached stabilizer in patients with Billroth II gastrectomy
}

Because a conventional sphincterotome is designed for an orientation of 11-12 o'clock, it is challenging during endoscopic sphincterotomy (EST) to incise the papilla hood in the ideal position (5-6 o'clock) from an inverted position in patients with Billroth II (BII) gastrectomy [1 -4]. After obtaining written informed consent, we attempted EST on seven consecutive patients with bile duct stones, who had previously undergone BII gastrectomy. Patient details are given in 0 Table 1 ; mean age was 74.4 (range $47-85$ years).

We attempted EST using a pull-type sphincterotome with an attached stabilizer (KD-19Q; Olympus, Tokyo, Japan) in conjunction with a standard forwardviewing endoscope (GIF-Q260; Olympus, Tokyo, Japan) ( $\bullet$ Fig. 1).

After accessing the duodenal stump and straightening the scope with a pulling maneuver, we identified the main papilla near the 12 o'clock position in the visual field with the tip of the scope oriented upward ( $\bullet$ Fig. 2).

When the downward-bending sphincterotome that has been forced into a sigmoid shape in advance is inserted through the scope, the cutting wire is usually directed downward along the upward-oriented tip of the endoscope ( Fig. 1). Using this technique, we successfully performed ESTs and stone extractions in all cases ( $\bullet$ Figs. 2 and 3). There were no adverse events during or after the procedures.

This technique has several advantages. Because an elevator is not required, we can select a forward-viewing scope, which can more easily be navigated to the papilla, instead of a side-viewing duodeno-

Table 1 Patient characteristics.

\begin{tabular}{|c|c|}
\hline Characteristic & No. of patients \\
\hline \multicolumn{2}{|l|}{ Gender } \\
\hline Male & 5 \\
\hline Female & 2 \\
\hline \multicolumn{2}{|l|}{ Stone size } \\
\hline$<10 \mathrm{~mm}$ & 3 \\
\hline$\geq 10 \mathrm{~mm}$ & 4 \\
\hline \multicolumn{2}{|l|}{ No. of stones } \\
\hline$\leq 2$ & 5 \\
\hline$\geq 3$ & 2 \\
\hline
\end{tabular}
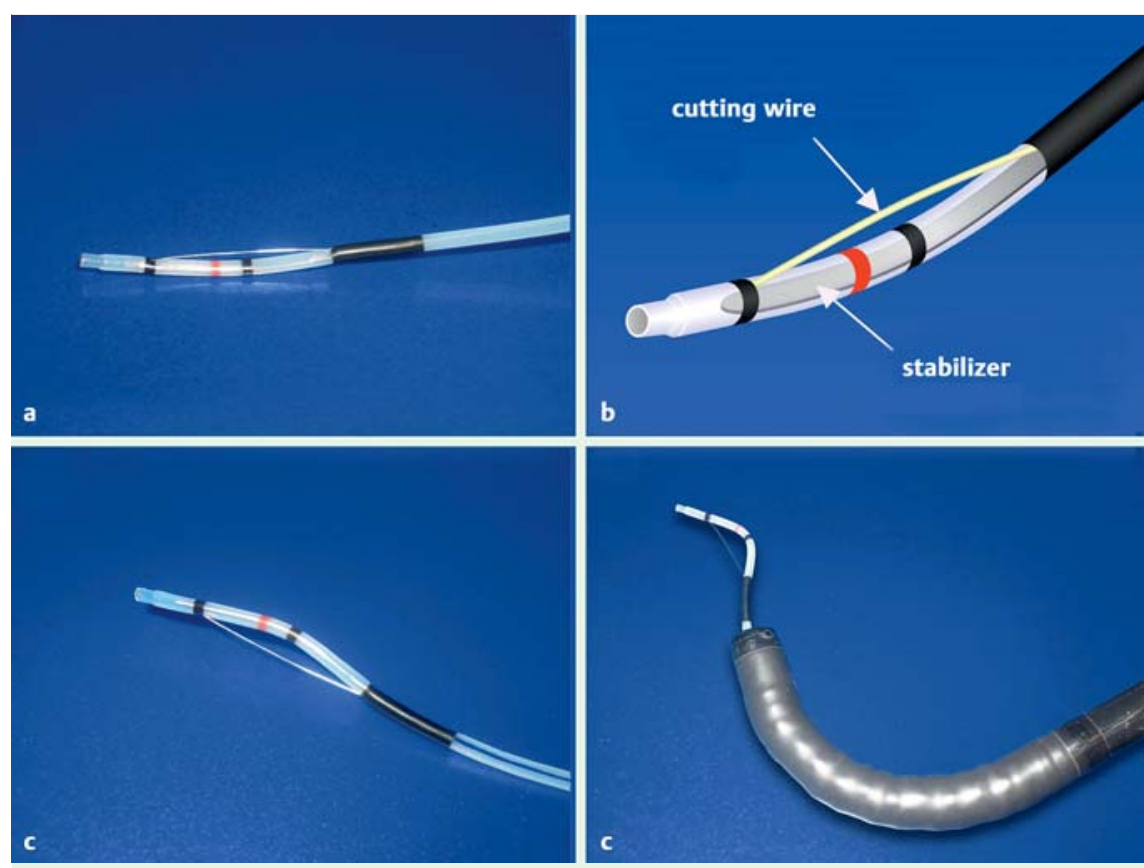

Fig. 1 a, b Pull-type sphincterotome with a stabilizer at the tip. c Bending the stabilizer downward at the tip and pulling the wire into the catheter forces the tip to form a sigmoid curve. $\mathbf{d}$ The cutting wire of the sphincterotome is directed downward through a standard forward-viewing endoscope when the scope is flexed upward.
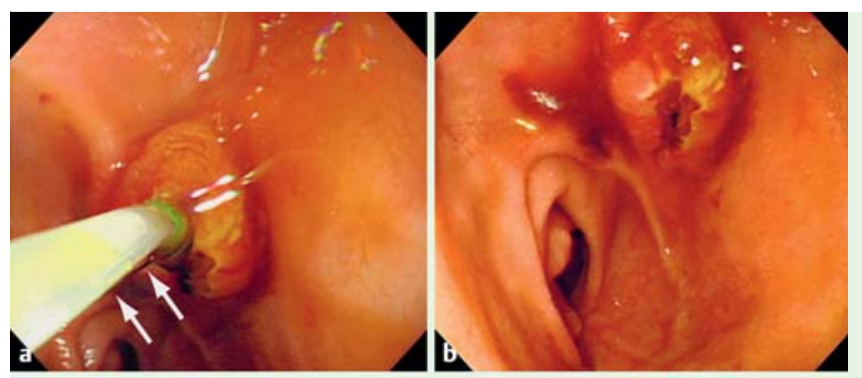

Fig. 2 a Endoscopic findings after reaching the papilla. The main papilla is identified near the 12 o'clock position of the duodenum. Endoscopic sphincterotomy (EST) is attempted under direct vision using this technique. (Arrows indicate the
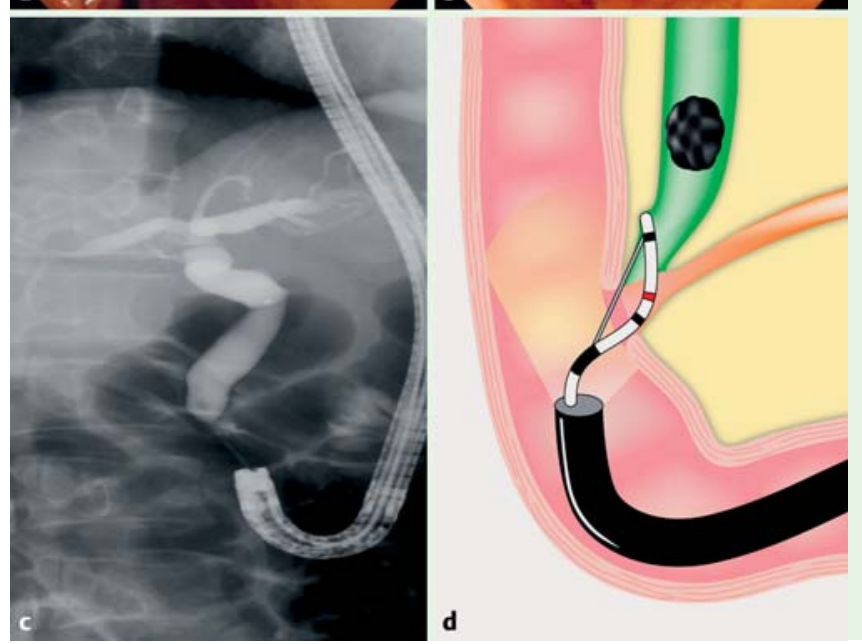
duct is opened by this technique. c Fluoroscopic findings during endoscopic retrograde cholangiopancreatography. Cholangiography demonstrated a stone in the bile duct. $\mathbf{d}$ Schematic representation of this technique. 


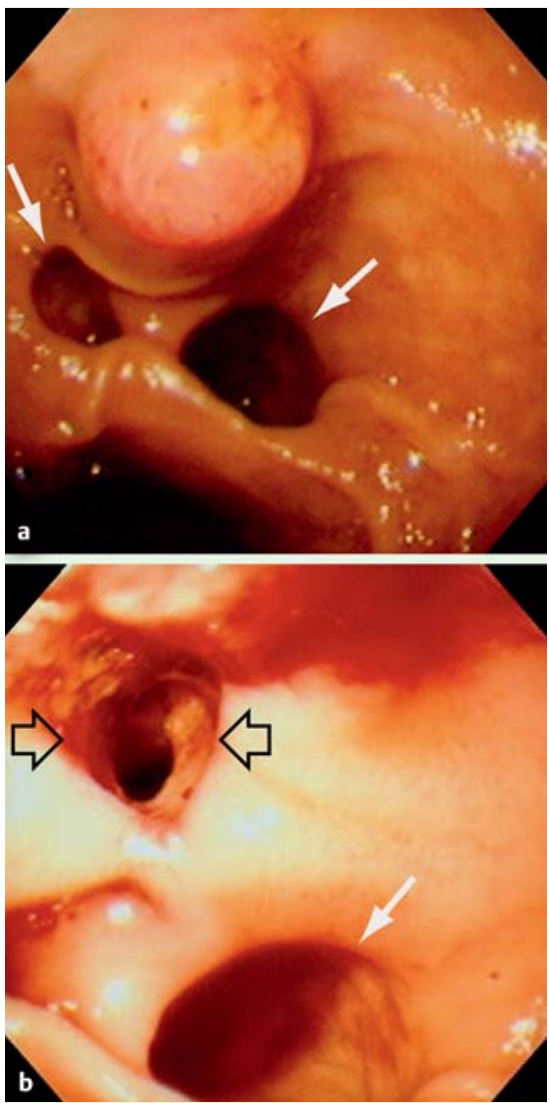

Fig. 3 The papilla of Vater in a patient with Billroth II gastrectomy $\mathbf{a}$ before and $\mathbf{b}$ after endoscopic sphincterotomy (EST). Periampullary diverticula (solid white arrows) did not have any significant negative influence on the procedure when using this technique. (Black arrows indicate the papilla opened by this technique.)

scope with an elevator [5]. In addition, the cutting wire can easily be fixed to the axis of the bile duct, and incision can be performed at the ideal position on the papilla under direct visualization ( $\bullet$ Fig. 2).
Table 2 Diagnostic and therapeutic findings.

\begin{tabular}{|l|l|}
\hline & No. of patients \\
\hline $\begin{array}{l}\text { Periampullary diverticula } \\
\text { Yes }\end{array}$ & 3 \\
No & 4 \\
\hline Braun anastomosis & \\
Yes & 2 \\
No & 5 \\
\hline Number of treatments & \\
for complete stone & \\
extraction* & \\
One & \\
Two & 4 \\
\hline Incision size & 3 \\
\hline Small & \\
Medium & 0 \\
Large & 2 \\
\hline Complications & 5 \\
\hline Yes & \\
\hline No & 0 \\
\hline
\end{tabular}

${ }^{*}$ At the first treatment session, we finished the therapeutic procedures by performing a biliary drainage such as endoscopic retrograde biliary drainage. In later sessions, we attempted endoscopic sphincterotomy (EST) to remove bile duct stones. Therefore, the first treatment session for drainage alone was excluded from the number of treatment sessions.

In these cases, we successfully performed ESTs using this technique. Table 2 gives the diagnostic and therapeutic findings; the mean procedure time was 59.5 minutes (range 40-90 minutes). However, this evaluation is limited to a small number of patients, and a larger study is required in the future.

\section{Endoscopy_UCTN_Code_TTT_1AR_2AC}

Competing interests: None

\section{K. Fujita', S. Myojo², S. Yoshida²,}

\section{Y. Kawase ${ }^{3}$}

1 Department of Gastroenterology, Meimai Central Hospital, Akashi, Japan

2 Department of Gastroenterology and Hepatology, Tabata Gastrointestinal Hospital, Akashi, Japan

3 Department of Internal Medicine, Kawase Clinic, Shiso, Japan

\section{References}

1 Van Buuren HR, Boender J, Nix GA, van Blankenstein $M$. Needle-knife sphincterotomy guided by a biliary endoprosthesis in Billroth 2 gastrectomy patients. Endoscopy 1995; 27: 229-232

2 Rosseland AR, Osnes M, Kruse A. Endoscopic sphincterotomy (EST) in patients with Billroth II gastrectomy. Endoscopy 1981; 13: $19-24$

3 Soehendra N, Kempeneers I, Reynders-Frederix $V$. Ein neues Papillotom fur den Billroth-II Magen. Dtsch Med Wochenschr 1980; 105: $362-363$

4 Hintze RE, Veltzke W, Adler A et al. Endoscopic sphincterotomy using an S-shaped sphincterotome in patients with a Billroth 2 or Roux-en-Y gastrojejunostomy. Endoscopy 1997; 29: $74-78$

$5 \mathrm{Kim}$ MH, Lee SK, Lee MH et al. Endoscopic retrograde cholangiopancreatography and needle-knife sphincterotomy in patients with Billroth II gastrectomy: a comparative study of the forward-viewing endoscope and the side-viewing duodenoscope. Endoscopy 1997; 29: $82-85$

\section{Bibliography}

Dol $10.1055 / \mathrm{s}-0030-1256082$

Endoscopy 2011; 43: E47-E48

(c) Georg Thieme Verlag KG Stuttgart · New York . ISSN 0013-726X

\section{Corresponding author}

\section{K. Fujita, MD}

Department of Gastroenterology

Meimai Central Hospital

Matsugaoka 4-1-32

Akashi

Hyogo 673-0862

Japan

Fax: +81-78-9141877

001579fujita@meimai-c-hp.com 